\title{
Response of Motor Vehicles, Front and Rear Axles of Which Are Fitted with Different Tires. Main Aspects
}

\author{
Vladimir Makarov ${ }^{1}$, Yuriy Molev 1,*, Dimitriy Proshin ${ }^{1}$, and Maksim Cherevastov ${ }^{2}$ \\ ${ }^{1}$ Nizhny Novgorod State Technical University named after R.Ye. Alekseev, Russia. \\ ${ }^{2}$ Minin Nizhny Novgorod State Pedagogical University, Russia.
}

\begin{abstract}
This work shows the modelling of change of such a controllability indicator as the stabilization time, using the design model of a vehicle with tires of different stiffness on the front and rear axles. The influence of the change in the lateral slip resistance coefficient of the front and rear wheels of the vehicle on the safe speed at which the stabilization reaction of the vehicle to the steering wheel turn will be aperiodic monotonic rather than oscillating, is established. The dependences of the magnitude of the stabilization time on the degree of change in the lateral slip resistance coefficient of the front and rear wheels, are obtained. The limiting values of the change in the parameters of the wheels providing the specified parameters of controllability which ensure acceptable road traffic safety level, are established. It is established that the maximum impact on the deterioration of the response of vehicle is exerted by the wheels design. On the other hand, their geometric dimensions have little to no road traffic safety impact. Based on the research carried out, it is necessary to conclude that in order to ensure road traffic safety, it is necessary to stop using the tires with different designs on different axles of the vehicle. Herewith, the pressure in the tires of the vehicle must not be less than the values set by the manufacturer. The results obtained are of interest to employees of institutions monitoring traffic indicators and technical condition of vehicles as well as carrying out road accident reviews.
\end{abstract}

\section{Introduction}

One of the main indicators of vehicle safety is its ability to move in the direction given by the driver, called the response of vehicle [1]. The control signal for changing the trajectory of the vehicle is the steering wheel angle. The consequence of changing the position of the steering wheel is a change in the driving parameters [2]. At the same time, due to the design features of the vehicle, the process of changing the driving parameters does not occur instantly. The process of changing the driving parameters during the transition from one steady state of motion to another is commonly called a stabilization process. Thus, the duration of the process of changing the driving parameters is the most convenient way to assess the response of vehicle, since this parameter has a physical meaning, a measurement

\footnotetext{
${ }^{*}$ Corresponding author: moleff@yandex.ru
} 
technique and directly affects the response of vehicle. According to the latest regulatory documents establishing the maximum time for the duration of the stabilization process, this value should be no more than 0.3 seconds for vehicles of categories M1, M2 and N1 and 2.0 seconds for vehicles of categories N2, N3 and M3, with lateral accelerations from 2 to 4 $\mathrm{m} / \mathrm{s}^{2}[3]$.

Vehicles put into operation are certified on the basis of compliance with the specified requirements as well. However, official vehicle testing does not imply that vehicles are fitted with tires of different designs. The following requirements apply to vehicles in service in terms of tire response ${ }^{\dagger}$ :

- $\quad$ spiked tires, if used, must be fitted to all wheels of the vehicle;

- winter tires, if used, must be fitted to all wheels of the vehicle;

- it is not allowed to install the following tires on one axle of the vehicle - tires of different dimensions, designs (radial, diagonal, tubed, tubeless), with different speed categories, bearing capacity indices, tread patterns, winter and non-winter, new and refurbished, new and with a deepened tread pattern;

- it is not allowed to use tires refurbished by applying a new tread, on the front axle of the vehicle;

- repeated retreading of tires with a previously reconstructed tread is not allowed;

- retreading of tires aged seven years or older is not allowed.

Analysis of these requirements shows that the installation of tires of different dimensions, designs (radial, diagonal, tubed, tubeless), with different speed categories, bearing capacity indices, tread patterns, summer and all-season, new and retreaded, new and with a deepened tire tread pattern is possible on different axles of a vehicle. That is, the regulatory documents in force allow the installation of wheels with different values of the lateral slip resistance coefficient on the front and rear tires, which will certainly affect the response of vehicles. Thus, further development of road transport, including the improvement of its safety, will undoubtedly be associated with the development of requirements for the response of tires [4].

\section{Research Methods}

To determine the degree of influence of the difference in the design of tires installed on the front and rear axles of the vehicle, a flat single-mass model of the vehicle is used, which, with its sufficient simplicity, allows obtaining reliable results, the accuracy of which is sufficient to solve the problem. [5]. In this context, the design model is described by such structural parameters of the vehicle as mass $-M[\mathrm{~kg}]$, moment of inertia $-J\left[\mathrm{~kg} \cdot \mathrm{m}^{2}\right]$ relative to the vertical axis passing through its center of gravity, wheelbase value $-L[\mathrm{~m}]$, distance (horizontal) from the center of gravity to the front axle $-a[\mathrm{~m}]$; distance (horizontal) from the center of gravity to the rear axle $-b[\mathrm{~m}], \zeta_{1}, \zeta_{2}-$ lateral slip resistance coefficients of the front and rear axles of the vehicle $[\mathrm{rad} / \mathrm{N}], \theta$ - steering angle $[\mathrm{rad}] ; \omega$ - turning rate of the vehicle $[1 / \mathrm{s}] ; s$ - Laplace operator; $C_{l}-$ front wheels cornering power $[\mathrm{N} / \mathrm{rad}] ; C_{2}-$ rear wheels cornering power [N/rad]; $V$ - travelling speed of the vehicle $[\mathrm{m} / \mathrm{s}]$. To assess the stability of the vehicle, the following indicators are used: $Y_{\beta}=C_{1}+C_{2}$ - coefficient of

\footnotetext{
${ }^{\dagger}$ Technical Regulations of the Customs Union on the safety of wheeled vehicles, TR CU 018/2011
} 
lateral force caused by wheel slip $[\mathrm{N} / \mathrm{rad}] ; Y_{\omega}=\frac{1}{V}\left(a C_{1}-b C_{2}\right)$ - coefficient of lateral force caused by the rotation of a vehicle relative to the vertical axis passing through its center of gravity $[\mathrm{N} \cdot \mathrm{s}] ; Y_{\theta}=-C_{1}$ - coefficient of lateral force caused by steering wheels [N/rad]; $N_{\beta}=a C_{1}-b C_{2}$ - coefficient of yaw moment caused by the wheel slip $[(\mathrm{N} \cdot \mathrm{m}) / \mathrm{rad}] ; N_{\omega}=\frac{1}{V}\left(a^{2} C_{1}+b^{2} C_{2}\right)$ - coefficient of yaw moment caused by the turning of a vehicle relative to the vertical axis passing through its center of gravity $[\mathrm{N} \cdot \mathrm{m} \cdot \mathrm{s}]$; $N_{\theta}=-a C_{1}$ - coefficient of yaw moment caused by steering wheels [(N.m)/rad].

\section{Results}

The stabilization response of the vehicle to steering wheel rotation can be both aperiodic monotonous and oscillating [5,6]. Moreover, the stabilization time in the first case is much less than in the second one. That is, from the point of view of road traffic safety, a process of the second type should be considered, in which the angular speed of the vehicle is described by the following equality [5]:

$$
\omega(t)=T_{1}+T_{4} e^{-\frac{B_{1}}{2} t} \sin \left(\omega_{k} t+\varphi_{k}\right)
$$

The travelling speed $V_{l}$, at which the stabilization response will be oscillating, is determined by the following expression:

$$
V_{1}=\sqrt{\frac{R_{1}-2 R_{5}}{2 R_{4}}}
$$

$$
\begin{gathered}
\text { where } R_{1}=-\frac{\left(a^{2} C_{1}+b^{2} C_{2}\right) M+\left(C_{1}+C_{2}\right) J_{z}}{M J_{z}} R_{4}=\frac{-a C_{1}\left(a C_{1}-b C_{2}\right) M}{J_{z}(a+b) C_{1} C_{2}} ; \\
R_{5}=\frac{-a C_{1}(a+b)}{J_{z}} .
\end{gathered}
$$

The relative change in the safe speed of the vehicle from the relative change in the stiffness of the front and rear wheels of the vehicle obtained by solving equation 2 , is shown in Figure 1.

According to experimental data, the amount of slip of modern wheels is in the range of values from $15 \ldots 40[\mathrm{kN} / \mathrm{rad}]$ for light vehicles to $60 \ldots 120[\mathrm{kN} / \mathrm{rad}]$ for motor trucks (according to the data specified in work [7]). That is, the real value of the change in the parameters of the lateral slip resistance coefficient of the wheels, depending on their design, can change by $1.5-1.7$ times, which results in the change of the safe speed of the vehicle by $20-30 \%$. A change in the lateral stiffness of the front axle in a wide range does not significantly affect the change in the duration of the stabilization process. At the same time, its value in the entire range of variation of the lateral slip resistance coefficient of axle, both upward and downward, is more than 0.3 seconds, which indicates an existing problem with ensuring the response of vehicle. The change in the lateral slip resistance coefficient of the rear axle most significantly affects the duration of the stabilization time, and when it 
increases by $10 \%$ relative to the initial value, a significant improvement in the response is observed. Thus, the lateral drift of the front axle in the studied range has a much smaller effect on the vehicle's handling than that of the rear axle. Reducing the stiffness of the rear axle results in a sharp increase in the stabilization time.

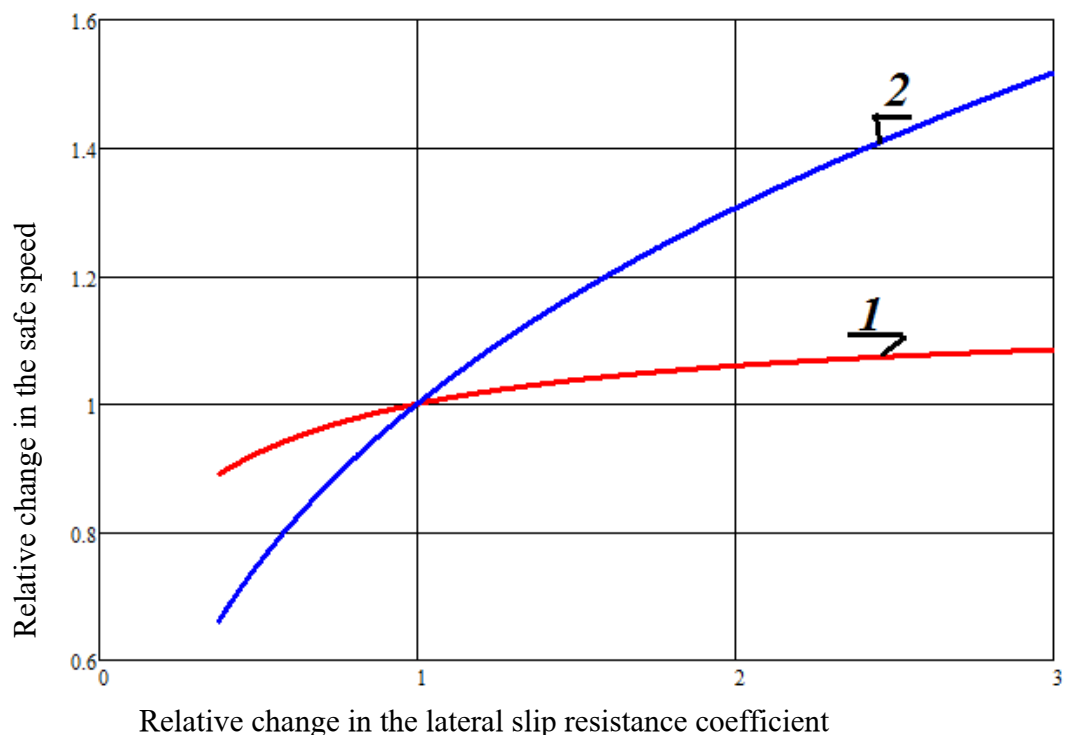

Fig. 1. Relative change in the safe speed of a fully loaded vehicle from the relative change in the lateral slip resistance coefficient of the front and rear wheels of the vehicle; 1 - for front axle wheels; 2 - for rear axle wheels

When the stabilization process proceeds according to the harmonic law, the following direct quality assessments [8,9] are of great practical interest: stabilization time or regulation time $\left(t_{p e z}\right)$; override $\sigma$, which is numerically equal to the following equation in percentage correlation: $\sigma=\frac{\omega_{\max }-\omega_{y c}}{\omega_{y c}} \cdot 100 \%$; number of oscillations during the stabilization process $n$.

To determine the time of the stabilization process, it is necessary to take into account that with $t>t_{p e c}$ the following condition is satisfied: $\left|\omega(t)-\omega_{y c}\right|<\Delta$. The value $\Delta$ is taken as $10 \%$ of the steady angular rate of rotation of the vehicle, which is equal to: $\Delta=\frac{1}{10} \omega_{y c}=\frac{1}{10} T_{1}$ or to: $\frac{1}{10} E_{1}$ depending on the nature of the process. Taking into account these refinements, calculations are made of the value of the time of the stabilization process at various values of $V$ and invariant $M$ and $\theta_{y c}$. In a graphical form, the dependence of the stabilization time for a rear-wheel drive motor truck of category N1 on the relative change in the lateral slip resistance coefficient is shown in Figures 2 and 3. 


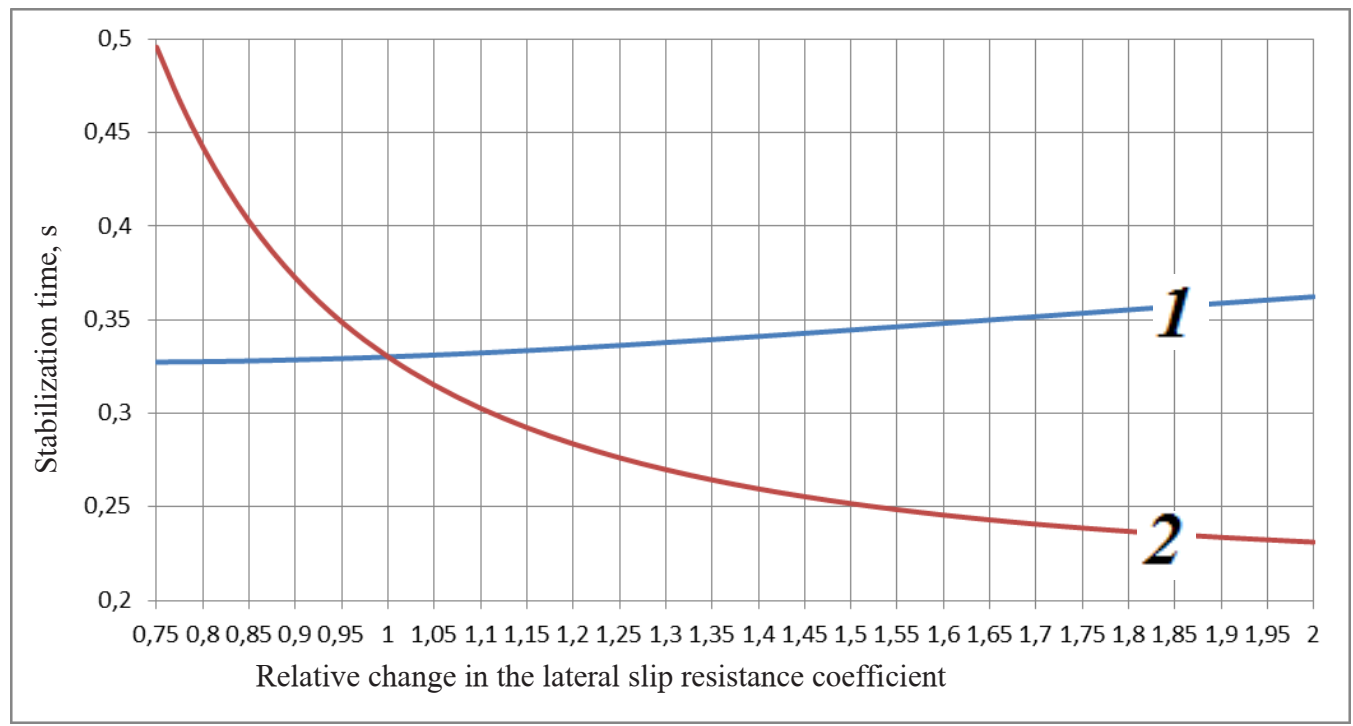

Fig. 2. Change in the stabilization time from the relative change in the lateral slip resistance coefficient of the front and rear wheels of the vehicle with a speed of $10 \mathrm{~m} / \mathrm{s} ; 1$ - for front axle wheels; 2 - for rear axle wheels

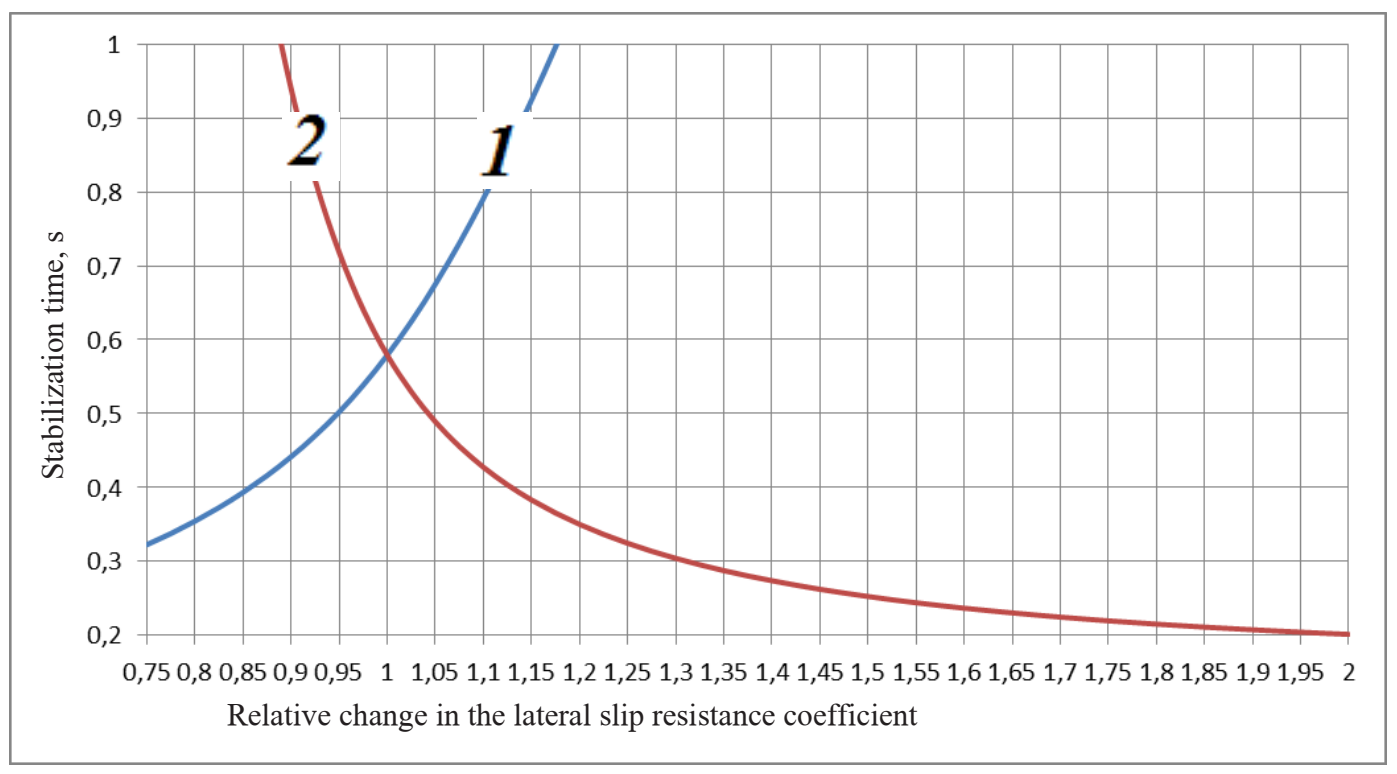

Fig. 3. Change in the stabilization time from the relative change in the lateral slip resistance coefficient of the front and rear wheels of the vehicle with a speed of $20 \mathrm{~m} / \mathrm{s} ; 1-$ for front axle wheels; 2 - for rear axle wheels

Summarizing the results obtained, it is possible conclude that the installation of wheels with increased lateral rigidity on the front axle is unacceptable from the point of view of safety. At the same time, a decrease in stiffness, having a positive effect on the response of vehicle in terms of the stabilization time, reduces the value of the speed at which the turning process begins to be oscillating. On the contrary, an increase in rear axle wheel 
stiffness by $30 \%$ can ensure normal response of the vehicle at full weight, and it increases the vehicle's safe corner entry speed.

According to the formula applied by A.Sh. Khusainov [10], the value of the lateral slip resistance coefficient can be determined from the following equation:

$$
C=-780 B_{\kappa}\left(d+2 B_{\kappa}\right)\left(p_{u}+98\right)
$$

where $B_{K}$ - tire width in meters, $d$-diameter at rim seat in meters, and $p_{u}$ - tire pressure in $\mathrm{kPa}$. Solving this equation, it can be noted that when the pressure in the tire drops by 0.3 atmospheres $(30 \mathrm{kPa})$, the value of the relative change in the lateral slip resistance coefficient will change by no more than $10 \%$, and the duration of the transient process will change by no more than 0.2 seconds. The results obtained were verified during field tests of vehicles manufactured by the Gorky Automobile Plant [11].

\section{Discussion and Conclusion}

The results obtained indicate that in order to ensure normal response of vehicle, the tire pressure should not be less than the value set by the manufacturer. The operational width of the wheels and their diameter at rim seat, allowed by manufacturers, have a less significant effect on the response of vehicle. Thus, a $5 \%$ reduction in the wheel profile width (which is permissible from the manufacturer's point of view) leads to a change in the lateral slip resistance coefficient by no more than $3 \%$, and the stabilization time will change by no more than 0.05 seconds. Therefore, tires of the same design, but with a different diameter at rim seat and profile width, can be used on the front and rear axles of the vehicle.

On the contrary, it should be noted that tires of different designs, but of the same standard size, could have a difference in the value of the relative change in the lateral slip resistance coefficient equal to $30 \%$ [7]. Such a spread in the tire stiffness values can lead to a decrease in safe driving speed by $90 \%$, and in the stabilization time - up to 2 seconds, which is unacceptable from the point of view of the response of vehicle. Since the value of the lateral slip resistance coefficient is not indicated by the manufacturers of tires, and its values are obtained experimentally, it is necessary to stop using tires with different designs on different axles of the vehicle to ensure road safety. The results obtained will not only improve road traffic safety, especially when vehicles move at speeds above $40 \mathrm{~km} / \mathrm{h}$, but also increase fuel efficiency [12].

The results of the given study have been obtained with the financial support of the Grant of the President of the Russian Federation No. MD-226.2020.8.

\section{References}

1. Litvinov A.S. Controllability and stability of the car - M.: Mashinostroyenie Publ., (1971).

2. Ni J., Hu JB. Dynamic modelling and experimental validation of a skid-steered vehicle in the pivotal steering condition//Proceedings of the Institution of Mechanical Engineers, Part D: Journal of Automobile Engineering. Volume: 231 Issue: 2 Pages: 225-240 (2017).

3. Gunzburg L.L., Nosenkov M.A. On the question of optimal response time of the car to control [Text]. / Controllability of cars and road trains. Steering drive. Trudy NAMI [Works by NAMI]. Issue 129. M.: (1971). 
4. Vantsevich V. Road and off-road vehicle system dynamics. Understanding the future from the past// Vehicle System Dynamics. 2015, No. 2, p.137-153.

5. Molev Y., Cherevastov M., Erasov I., Levshunov L. Indirect quality estimates of the vehicle movement response to the control step input/ IOP Journal of Physics: Conference Series. C. 012029 (2019).

6. Gray JP., Vantsevich V., Paldan J. Agile tire slippage dynamics for radical enhancement of vehicle mobility // Journal of Terramechanics. Volume: 65 p/pp.14-37 (2016).

7. Yuryev Yu. Research of resistance coefficients to lateral tire slip: Dis. of Cand. tech. sciences. M., (1970).

8. Vakhidov U., Molev Yu., Sogin A., Slyusarev A., Cherevastov M. Direct assessments of the reaction quality of the car movement to the step control action / Journal of Proceedings of NSTU named after R.E. Alekseev, No. 3 (122), (2018).

9. Belyakov V., Kurkin A., Makarov V., Zeziulin D. Multifunctional vehicle for coastal areas / The Twelfth International Conference on the Mediterranean Coastal Environment (MEDCOAST 2015) 06-10 October 2015, Varna, Bulgaria p.945-951 (2015).

10. Khusainov A., Operational properties of the car: textbook. - Ulyanovsk: UITU, (2011).

11. Makarov V., Korelin O., Koblyakov D., Kostin S., Komandirov A. Development of driver's assistant system of additional visual information of blind areas for Gazelle Next/ Paper presented at the IOP Conference Series: Materials Science and Engineering, 315(1), (2018).

12. Makarov V., Zezyulin D., Maleev S., Ogorodnov S. Calculation method of vehicles fuel consumption during movement along a route/ Proceedings of 3rd Asian Pacific Conference on Mechatronics and Control Engineering (APCMCE 2015), pp. 306-310 (2015). 